\title{
The effects of threat and attraction on interpersonal bargaining'
}

\author{
LOUIS TORNATZKY and P. JAMES GEIWITZ, Stanford \\ University, Stanford, Calif. 94305
}

This study attempted to clarify the conditions in which threats are conducive to cooperation. Pairs of Ss in a Prisoner's Dilemma game either had the opportunity to send threat and penalty messages, or they did not. In addition, pairs were either of high or low mutual positive attraction, manipulated by perceived attitude similarity. In terms of the number of cooperative responses in the game, the high mutual attraction conditions showed significantly more cooperative behavior than those groups of low mutual attraction. A significant interaction effect was demonstrated: Over blocks of trials the efficacy of threats and penalties in inducing cooperation was contingent upon whether the Ss were of high or low mutual attraction. As the game progressed the no-threat condition produced more cooperation than the threat/penalty condition providing high mutual attraction was present; with low mutual attraction, the threat/penalty condition was increasingly superior to the no-threat condition. In general, the beneficial effect of threats in low attraction dyads was not observable in high attraction pairs. These results were interpreted as reflecting the incongruity of threats and penalties in a "friendly" interaction.

Since the work of Deutsch \& Krauss (1962), the effect of threats on cooperation has been a crucial issue in the study of interpersonal bargaining. These investigators used "gates" as threats in a trucking game and found that they decreased interpersonal coordination. In contradiction, Shomer, Davis, \& Kelley (1966) used a modified trucking game and found the presence of "financial" threats to increase cooperation. Much of the theoretical and empirical controversy is in how threats are perceived and used. Deutsch \& Krauss see the use of threats as a punitive and hostile aspect of the interaction and thus detrimental to cooperative interchange, while Shomer et al see them as a signalling device with which members of a dyad can coordinate moves and reach cooperation.

In an effort to clarify some of these issues and to provide a more controlled and simpler experimental game, Geiwitz (1967) used the Prisoner's Dilemma (PD) in which some pairs of Ss were able to send explicit messages in the form of threats of financial penalties. The presence of threats served to increase cooperative behavior compared with conditions in which this message-sending capacity was absent. In a later study (Geiwitz \& Fisher, 1968), it was demonstrated that this facilitating effect was due to the explicit signalling and implicit deterrent aspects of the threats.

It seems a reasonable summary of available research to say that the effects of threats are highly dependent on the context in which they are delivered. Heretofore, interest has focused on the primary contextual features of threat form (e.g., gates and financial threats) and situation (trucking game with or without the alternative route and PD). While elaboration of these influences remains an essential task, contextual features of other types also require study. In this experiment, the effect of high or low "liking" for the other player will be examined.

Research by Krauss (1966), Swingle (1966), and Kaufmann (1967) has shown that interpersonal attraction has a beneficial effect on bargaining. But it is by no means an unequivocal prediction to state that attraction will enhance cooperation even more when threats are present. even if threats are in a form and situation shown to be beneficial for anonymous opponents, as is the case here. They may do so because of their explicit communication potential, but, on the other hand, they may be perceived as incongruous, annoying, and disruptive in a friendly interaction, and hence decrease cooperation. In dyads of low mutual attraction, threats may be beneficial because of "warranted" communication or they may be detrimental because of the greater possibility of hostility in such pairs. This experiment was designed to answer these questions.

\section{METHOD}

The Ss were 80 male high school students recruited for a series of experiments by an advertisement in a local newspaper. They were paid $\$ 1.50$ for their participation, plus winnings acquired during the game. The game was a Prisoner's Dilemma with matrix values of 10 points each for mutual cooperation, -10 for mutual competition, and 15 and -15 for "competitor" and "cooperator," respectively, in the other two cells. The matrix was printed on an electronic game board with push buttons to indicate choices and, for half the Ss, switches for sending standard threats ("I am considering using the 10\% penalty") and standard penalties ("I make you lose $10 \%$ of earnings").

The Ss were run in groups of approximately 20 . They were visually shielded from the other member of their dyad by a high table divider. Recorded instructions described the simple mechanics of the game and the nature of the dilemma. Ss were told that the points to be won or lost were to be converted to cash at the end of the experiment "at a predetermined rate." Although that rate was one cent for every 10 points, Ss commonly assumed they were playing for a penny a point.

One half of the pairs then received written instructions on the nature and mechanics of threat and penalty statements. Threats and penalties were bilateral, applied to total earnings at the point of transmission and did not. accrue to the "penalizer"; penalties did not have to follow threats, nor did Ss have to precede a penalty with a threat; one message was allowed between any two of the 18 trials of the game. (To this

Table 1

Means and Analysis of Variance in Cooperative Responses

\begin{tabular}{lcccc}
\hline & \multicolumn{4}{c}{ Btocks of Six Trials $(\mathrm{N}=6$ per block) } \\
\cline { 2 - 6 } Condition & 1 & 2 & 3 & overall \\
\hline HT & 4.70 & 4.70 & 4.95 & 4.78 \\
HNT & 4.50 & 4.95 & 5.45 & 4.97 \\
LT & 4.75 & 4.25 & 4.50 & 4.50 \\
LNT & 4.35 & 3.35 & 2.60 & 3.43 \\
overall & 4.58 & 4.31 & 4.38 & \\
\hline Source of Variance & & df & MS & F \\
\hline A (Threat) & 1 & 11.704 & N.S. \\
B (Attraction) & 1 & 49.504 & $5.979^{b}$ \\
AB & 1 & 23.438 & $2.831^{\mathrm{a}}$ \\
AB x Ss within & groups & 76 & 8.279 & \\
C (Blocks) & 2 & 1.504 & N.S. \\
AC & 2 & 1.004 & N.S. \\
BC & 2 & 13.004 & $10.219^{c}$ \\
ABC & 2 & 6.088 & $4.784^{b}$ \\
ABC x Ss within groups & 152 & 1.273 & \\
\hline
\end{tabular}

$a_{p}<.10$

$b_{p}<.05$

$c p<.01$ 
point, the apparatus and procedure are essentially identical to that used by Geiwitz \& Fisher, 1968, where the reader will find a more detailed description.)

We have defined one of the independent variables as availability of threat-penalty (T) or not (NT). Then the second manipulation was introduced by supplying each $S$ with an attitude questionnaire supposedly (but not in fact) completed by his opponent. Ss had themselves completed this questionnaire earlier and now received one that was either in $100 \%$ or $0 \%$ agreement with their opinions. Byrne $(1961)$ has used this procedure in a number of experiments to produce different degrees of interpersonal attraction, in our case either high (H) or low (L). As a check on the manipulation, Ss then were given Byrne's Interpersonal Judgment Scale (IJS).

The four conditions, with 20 Ss randomly assigned per condition, were thus HT (high mutual attraction, threats and penalties available), HNT, LT, and LNT.

\section{RESULTS}

To test the effectiveness of the attitude similarity manipulation, the IJS ratings of the $H$ groups were compared to those of $\mathrm{L}$ groups. The mean rating of 9.74 for $\mathrm{L}$ was significantly different from the 4.78 for $H \quad(t=5.05$, $p<.001$ ), with lower numbers indicating greater liking on a scale ranging from 2 to 14 .

Table 1 gives the mean number of cooperative choices for each condition over three blocks of trials (six trials per block) and the analysis of variance for these results. Dyads of high mutual attraction choose the cooperative response significantly more often than dyads of low mutual attraction. High Attraction Ss tend to increase in cooperativeness while low Ss tend to decrease. While the result does not quite reach acceptable levels of significance, threats empirically have the result of improving cooperation for low attraction dyads and are detrimental for high attraction dyads. The significant triple interaction is caused by an increasingly superior performance by NT Ss in $\mathrm{H}$ pairs and an increasingly inferior performance by NT Ss in L pairs.

\section{DISCUSSION}

The availability of threats of this type is an advantage in bargaining between Ss who do not like one another, just as they are advantageous in dyads of anonymous players (Geiwitz, 1967). They do not appear to be advantageous to pairs of high mutual attraction; indeed, they are slightly detrimental. In low attraction dyads, we could assume that threats function somewhat similarly to the way they influence cooperation in anonymous pairs (Geiwitz \& Fisher, 1968). When actually used, they serve as cooperative signals, and their mere presence deters players from whimsical or hostile attempts at exploitation in the PD. In high attraction pairs, however, the presence of threats is not "in balance" with the friendly relationship. One does not expect a threat or penalty from a friend and when used, they may be perceived as unnecessary, unwarranted, and hence detrimental to the "friendship" and the interaction. Even the presence of such messages may cast an air of uncertainty to an interaction that is typically based on confidence: confidence in trust, mutual support, and expectations of supportiveness, generosity, and cooperativeness.

\section{REFERENCES}

BYRNE, D. Interpersonal attraction and attitude similarity. Journal of Abnormal and Social Psychology, 1961, 62, 713-715.

DEUTSCH, M., \& KRAUSS, R. M. Studies of interpersonal bargaining. Journal of Conflict Resolution, 1962, 6, 52-76.

GEIWITZ, P. J. The effects of threats on prisoner's dilemma. Behavioral Science, 1967, 12, 232-233.

GEIWITZ, P. J. \& FISHER, S. J. Threats, statements of intention, and interpersonal conflict. Unpublished paper, Department of Psychology, Stanford University, Stanford, California, 1968.

KAUFMANN, H. Similarity and cooperation received as determinants of cooperation rendered. Psychonomic Science, 1967, 9, 73-74.

KRAUSS, R. M. Structural and attitudinal factors in interpersonal bargaining. Journal of Experimental Social Psychology, 1966, 2, 42-55.

SHOMER, R. W., DAVIS, A. H., \& KELLEY, H. H. Threats and the development of coordination: Further studies of the Deutsch and Krauss trucking game. Journal of Personality \& Social Psychology, $1966,4,119-126$.

SWINGLE, P. G. Effects of the emotional relationship between protagonists in a two-person game. Journal of Personality \& Social Psychology, 1966, 4, 270-279.

NOTE

1. This research was supported by a grant from the National Science Foundation, GS-1680, to the junior author. 